$\mathrm{d} \ln J_{2} / \mathrm{d} V$ are in better agreement with the experiment.

The author should like to thank Dr. H. Billing and Dr. G.Heldmann for helpful discussions.

\section{References}

1. J.G.Simmons, Brit. J. Appl. Phys. 18 (1967) 269.

2. K.H.Gundlach, Proc. Int. Symp. on Basic problems in thin film physics, Clausthal-Göttingen 1965, eds. H. Mayer and R. Niedermayer (Vandenhoeck and Ruprecht, Göttingen. 1966). p. 696.
3. S.R. Pollack and C.E.Morris, J. Appl. Phys. 35 (1964) 1503.

4. S.R. Pollack and C.E.Morris, Trans. Met. Soc. AIME 233 (1965) 497

5. Z. Hurych. Solid-St. Electron. 9 (1966) 967.

6. W.A.Harrison, Phys. Rev, 123 (1961) 85.

7. W. Franz, in Handbuch der Physik, ed. S. Flügge (Verlag Julius Springer. Berlin, 1956) Vol. XVII. p. 155 .

\title{
ELECTRON SPIN POLARIZATION IN FIELD EMISSION FROM Gd
}

\author{
N. MÜLLER and H. Chr. SIEGMANN \\ Sektion Physik der Universität Minchen \\ and \\ G. OBERMAIR \\ Theoretisches Teilinstitut, Physik Department der Techn. Hochschule, Minchen
}

Received 15 May 1967

\begin{abstract}
Theoretical considerations of the conduction electron polarization in Gd are reported. They predict that $5-10 \%$ polarization ought to be measured in an electron beam produced by field emission.
\end{abstract}

The electron current from a field emission cathode is produced by tunneling from the electron states at and directly below the Fermi level within some $10^{-2} \mathrm{eV}[1]$.

A field cathode, therefore, could be an intense source of polarized electrons, if one could produce sufficient electronic spin polarization at the Fermi edge and if this polarization remained unaffected by surface effects in the tunneling. Polarization at Fermi level $E_{\mathrm{F}}$ can be obtained in two ways: paramagnetic material in very high fields at very low temperatures shows the desired effect ("brute force method"); the obtainable difference in spin population at $E_{F}$, however, is extremely small [2] in most metals and semiconductors. Only a narrow gap semiconductor with very small Fermi energy in the conduction band $\left(m^{*} \ll m\right)$ like InSb can be regarded as a hopeful candidate [3].

In ferromagnets a certain spontaneous spin polarization at the Fermi level should exist. Against some theoretical predictions no electron beam polarization was found in the case of $\mathrm{Fe}$ and in photo emission from $\mathrm{Ni}$ (references in [2]).

The failure of these attempts in 3d-ferromagnets can be explained in two ways: a) Surface effects in field emission lead to a complete destruction of the polarization existing in the interior of the metal at Fermi level. b) The "effective" density of states, i.e. the density averaged over the respective contributions of $3 \mathrm{~d}$ and $4 \mathrm{~s}$ electrons to the emission current is the same for the two spin directions. This could occur, if the density of states $n(E)$ were practically constant over the energy interval $E_{\uparrow}-E_{\downarrow}$ around $\bar{E}_{\mathrm{F}}=\frac{1}{2}\left(E_{\uparrow}+E_{\downarrow}\right)$, where $E_{\uparrow}$ and $E_{\downarrow}$ are the effective Fermi levels for the two spin states, or if the $4 \mathrm{~s}$ electrons with their probably vanishing polarization predominate in the emission.

Assuming explanation b) to be true for Fe and Ni (for Co it should be the same, then) one can consider the situation in ferromagnetic Gd. Here we have the following facts:

a) The saturation magnetization is $7.55 \mu_{\mathrm{B}}$ per 
atom [4], of which $s=0.55$ have to be attributed to the conduction band, because the seven $4 \mathrm{f}$ electrons are localized and energetically at least 10 $\mathrm{eV}$ below the conduction band [5].

b) The total number of conduction electrons is $n=3$ per atom. Recent band structure calculations [5] show a mixture of $5 \mathrm{~d}$ and $6 \mathrm{~s}$ states for the conduction band. The results for the density of states up to the Fermi energy $\bar{E}_{F}$ of about $2.5 \mathrm{eV}$ can be approximated by a parabolic band with $m^{*}=$ $=3 m, 2 n\left(\bar{E}_{\mathrm{F}}\right)=1.8 \mathrm{eV}^{-1}$. We have then

$$
\begin{aligned}
& n=\int_{0}^{E_{\uparrow}} n(E) \mathrm{d} E+\int_{0}^{E_{\downarrow}} n(E) \mathrm{d} E, \\
& s=\int_{0}^{E_{\uparrow}} n(E) \mathrm{d} E-\int_{0}^{E_{\downarrow}} n(E) \mathrm{d} E .
\end{aligned}
$$

With $n(E) \sim \sqrt{E}$ this gives $E_{\uparrow} \approx 2.8 \mathrm{eV}, E_{\downarrow} \approx 2.2$ $\mathrm{eV}$. The difference should be equal to the average effective exchange energy $E_{\uparrow}-E_{\downarrow}=0.6 \mathrm{eV}=$ $=2 S J_{\text {sf }}$, where $2 S=7$ is the number of uncompensated $4 \mathrm{f}$ spins and $J_{\mathrm{sf}}$ the exchange integral between one $4 f$ and one conduction band state. The result agrees reasonably with different calculations [6], [7] which give $J_{\mathrm{sf}}=0.05-0.1 \mathrm{eV}$.

This order of magnitude for the $4 f-6 s$ exchange can also be inferred from recent experiments about the shift of the optical absorption edge in EuO at its ferromagnetic transition [8], they show that $2 S J_{\mathrm{sf}} \geqslant 0.25 \mathrm{eV}$.

All these considerations and numbers fit together nicely within $50 \%$ uncertainty; inserted in the parabolic band approximation $n(E) \sim \sqrt{E}$ they predict a polarization of the conduction electrons at $\bar{E}_{\mathbf{F}}$ of

$$
p=\frac{n\left(E_{\uparrow}\right)-n\left(E_{\downarrow}\right)}{n\left(E_{\uparrow}\right)+n\left(E_{\downarrow}\right)}=6 \%
$$

If $n(E)$ varies more rapidly near $\bar{E}_{\mathrm{F}}, p$ can take on both larger and smaller values; small amounts of metals of different valency added to Gd could be used to investigate this behaviour and to increase $p$.

Based on these considerations we made a first experimental attempt to measure the polarization of field electrons from Gd [9]; for technical reasons this experiment did not succeed.

Meanwhile an apparatus of the type described in [10] was available. The promising experimental results will be published soon [11].

We are indebted to Dr. P.J. Kennedy and Dr. P.S. Farago for valuable discussions.

\section{References}

1. R.H.Good and E.W. Müller, Encyclopedia of Physics 21 (Berlin 1956).

2. P.S.Farago, Advan. in Electron Phys. 21 (1965) 1.

3. P.J.Kennedy, Phys. Letters 19 (1965) 161.

4. H. Nigh, S. Legvold and F.H.Spedding, Phys. Rev. 132 (1963) 1092

5. J.O.Dimmock and A.J.Freeman, Phys. Rev. Letters 13 (1964) 750.

6. T.A. Kaplan and D. H. Lyons, Phys. Rev. 129 (1963) 2092.

7. F. Milstein and L.B.Robinson, Phys. Rev. Letters 18 (1967) 308.

8. G. Busch and P. Wachter, Phys, kondens. Mat. 5 (1966) 232

9. N. Müller, Diplomarbeit Univ. München 1966, unpublished.

10. H. von Issendorff and R. Fleischmann, Z. Physik 167 (1962) 11.

at zero temperature. 